\title{
Commonplace
}

\section{Discomfort with Gold OA}

\author{
Ashley Jester
}

Published on: Apr 22, 2021

DOI: $10.21428 / 6 f f d 8432.913 a e f 43$

License: Creative Commons Attribution 4.0 International License (CC-BY 4.0). 
If you are someone like me, who keeps an eye on developments related to open access even (perhaps especially) when they don't affect you directly, you noticed that the University of California and Elsevier announced a pretty_big_deal, though not a Big Deal. It's a transformative agreement that the university administration says is delivering on its goals of making research from its affiliated faculty free for anyone in the world to read and reducing their projected budget costs. Whether the deal actually will deliver on these two points is under debate elsewhere, but I wish to focus on the part of the agreement that has been bothering me, not only about this particular deal but about all transformative agreements of this sort, from the very first signed by Carnegie Mellon in the halcyon days of 2019. The social scientist in me is willing to accept that the folks involved in the UC-Elsevier negotiations understand their own preferences and have represented them ably; by all accounts, this is a good deal for the UCs and for Elsevier. But the question I cannot stop asking myself is whether this is good for open access. And social science suggests it might not be.

In this essay, I'm going to make the case that open access agreements that rely upon a pay-to-publish model are good for the groups who sign them but bad for the overall system. To do that, I'll start with a quick refresher on public goods and then use that framework to discuss the state of open access and the variations in different colors as they relate to public goods. $\underline{1}$ After laying out a model of open access as a public good, I'll argue that what we are actually building is not a system rooted in public goods but one that has merely shifted the entry barriers from readers to authors. I'll conclude by offering some hope for the future, again rooted in the social science of public goods.

\section{Truly open or members only?}

Let me set up a framework for understanding open access today. Many people have heard of the "Tragedy of the Commons" or public goods, but we don't often get examples beyond things like, well, the commons or clean air and water. But public goods are anything that benefits everyone while conspicuously being in no one's individual interest to create or maintain. Why is that so? The same features that make public goods so useful -- they are non-excludable (everyone can use them!) and nonrivalrous (my using it doesn't impede your using it!) -- make them susceptible to exploitation.

Consider the classic example of the entrepreneur who builds a fence around open grazing ground and charges rents to farmers who had previously grazed their flocks for free. No one owned the land so no one stopped the fence-builder, and it's not clear 
which of the many farmers who used to graze their flock on the land wants to go through the trouble of dealing with getting the fence removed when it's just a few dollars every day to graze. And thus a public good gets converted into something else, in this case something called a club good. After the fence goes up, we can see the fence-builder getting some rents from the farmers, who are now enjoying less benefit from the grazing ground, and there are probably even more than a few farmers who have to give up altogether. The beauty of the public good, namely that no one owns it and can keep people from using it, also means that no one protects it and keeps people from abusing it.

If you think about the nature of open access, it meets the canonical requirements of a public good; when research is published openly, it's non-excludable (anyone can access it) and it's non-rivalrous (my consumption does not diminish yours). It's easy to see how open access is a public good from the point of view of the consumers of said research, but it's important to recognize that creating an open access ecosystem is not only about the reading side of the research transaction. Research must be published to be read, and it must be published openly to be read openly. Thus, open access, especially as a public good, is not only about the ability to read freely but about the ability to publish in openly accessible venues without paying for the privilege.

Much of the focus on open access has been centered on the ability to read freely, and this is understandable. The shift in the paradigm of access from a model where the reader pays to read research to one where the author pays to publish has been transformative. It is undeniable that providing open access in any flavor or color benefits consumers (i.e., readers) of research. While some forms of open access still impose significant transaction costs for the reader, especially in terms of search, one of the biggest barriers to access is removed with reading made free. My intent here is not to diminish the importance of reading for free but to emphasize that, in a truly public good fashion, publishing must also be free.

Before I go further, let me state clearly that I know publishing is not actually free. Economists tout the statement that "There is no free lunch," and this doesn't mean that you can't go somewhere and eat without paying but that food is not free. Publishing certainly entails real costs, not the least of which are the costs of hosting and supporting the digital infrastructure that modern academic research requires. $\underline{2}$ There are costs associated with creating the product -- the academic research -- and 
with refining and producing the final version, though these costs are quite often borne as the labor of researchers themselves who then donate this labor, uncompensated, to publishers. There are costs associated with distributing and maintaining the product, and, like any healthy industry, there are costs associated with growth and development and providing a reasonable return for the company's owners. If we believe that open access to academic research is a public good, we cannot make the creation and sustenance of that good depend upon the contributions of individual authors any more than we could make it work with individual readers. Public goods require collective solutions.

Public goods have that particular name for a reason, and the fact that governments are commonly associated with providing and protecting public goods fits with the nature of these goods as well. The entity that creates and sustains a public good bears more costs than it ever realizes in benefits; after all, when you've built something that you can't stop everyone else from using, it's simply impossible for you to realize all the benefits. $\underline{3}$ Your benefits look like everyone else's benefits, but your costs? Your costs are enormous and quite likely unsustainable. $\underline{4}$ This is the reason that public goods are, for the most part, supplied by governments and other entities (e.g., non-profit corporations or non-governmental organizations) that are large enough to bear the costs of creating and sustaining them and for whom creating a public good for which they do not extract the full economic value is part of their mission. $\underline{5}$ The mission of these groups is creating and sustaining a public good. They do this because they believe this good raises the overall value to the world; it's not just about taking care of themselves. This kind of mission is one that underpins many of the open access mandates generated by funders from governments to private agencies, namely the idea that research should be free for the world. For that to be real in the truest sense, it must be free not only to read but also to publish.

What worries me about the current state of things is that we -- and here I mean explicitly the largest universities and research institutions whose researchers are the most prolific at publishing and consuming academic research -- are building a system and enjoying the illusion of "free" open access while behind the scenes strengthening the idea that open academic research costs should be paid by authors of that research.

When we create a public-type good that depends upon individual contributions for its maintenance and sustenance, that's actually a club good. It's called a club good because the easiest examples of these types of goods are club memberships -- you join the golf club, and now you can get your membership's share of rounds without extra 
charge. Well-functioning clubs use the entry fees charged to members to create and sustain something that often looks like a public good, but it's not -- it's a club good. I'm guessing you might have had the experience of passing a beautifully landscaped property that looks like a public park but, when you get closer, is marked for members only. Gramercy Park in New York is a famous example, but there are countless others. What ties club goods together is their appearance and mimicry of a public good -- for members, the experience feels very much like a public good, and, if the club is large enough, it can even appear like a public good from a social standpoint. And of course, some clubs do advocate for issues in ways that create public good benefits -- think of the AARP lobbying for older Americans -- and blur the lines between what parts of the club are for members only and what parts serve as true public goods.

\section{Shifting the membership burden to authors}

Let us return to open access as a public good and look more closely and critically at how it's being supported. If we return to the specific example of the UC-Elsevier deal, it's easy to see the public goods benefit from the readers' perspective -- research that involves UC authors will be available without reading costs, and there are many researchers covered by this agreement which means that there will be a commensurate amount of scholarship opened to readers. The broad coverage and inclusivity of researchers in the UC system is not only a great benefit to readers but is also a clear benefit to researchers in the UC system. For those researchers who were previously unable to afford OA publishing, this agreement opens that door; for researchers who have been using their research funds to cover APCs, this agreement lowers their rates and bends their cost curves.

It's unclear whether the UC system will pay less overall under this new contract since the systemic costs depend upon just how much OA publishing happens, but it's clear that costs are lower under this contract than they would be without it, ceteris paribus. What is also clear is that this deal does not make it easier for anyone not affiliated with the UCs to publish in an Elsevier journal, ceteris paribus. While the deal creates a public good for readers, it provides a club good for the authors. You can get through the open access gate, but only if you are part of the UC club and even then, there may be still some fees associated if you have the money.

Of course, the UCs are not the only ones with a club. More and more agreements of these types are being signed; whether they are called read and publish or publish and 
read doesn't matter as much as the structure of the agreement wherein the covered group gets to publish their research with open access while retaining readership for the rest of the publisher's portfolio. Every deal like this one creates more opportunities for research to be published openly and increases the overall number of research works that are openly accessible. Yet, while these deals do create clear public goods for readers, they reinforce other parts of the system that are less desirable.
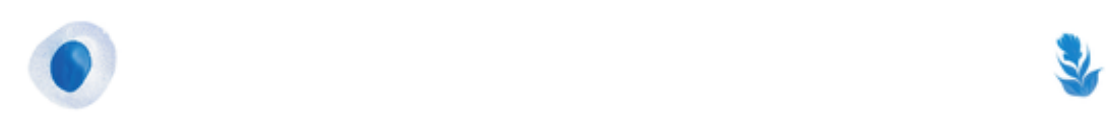

Transformative agreements that merge open access publishing with subscription-style readership further the idea that the authors of research should literally be paying the cost for an open access ecosystem. Placing the burden upon individual authors to pay for open access publishing makes the publishing side of research (distinct from the reading side) a club good. It's not something that any qualified researcher has access to by dint of the quality of their work; in order to be able to publish that work in an open access venue, you must pay some additional price. For researchers who cannot afford this entry fee, the set of choices dwindles quickly and considerably. When a deal is struck that covers some or all of the APCs, that deal explicitly validates the idea that APCs are an appropriate part of the open access ecosystem. You are no longer arguing about whether or not individual authors should have to pay to publish their work openly but are merely haggling over the terms. You have ceded the point that open access publishing should be free to anyone who produces research without an entry fee. These sorts of agreements do not lower the costs these researchers face to publishing their work openly. Researchers at smaller and less well-funded institutions already face an uphill battle in academia and academic publishing. $\underline{6}$ Again, club goods can look and feel like public goods, but they are not available to anyone on the wrong side of the gates.

Fundamentally, my discomfort with APC-based open access agreements like the one struck by the UCs and Elsevier is tied to my fear that the current system is headed toward a state where we attempt to create the public good of open access readership through the club good of open access publishing. In other words, I am worried that, by signing transformative agreements, we are agreeing in principle to a system in which individual pieces of research must be liberated one-by-one to create a giant pool of free scholarship for everyone to read. I have spent enough time studying public goods to know that you can't make them from clubs, and you can't build them by depending 
upon the motivations of individuals en masse. $\underline{7}$ While it looks like we are moving toward a more open system every time one of these agreements is signed, what I worry about is that we are really moving toward a system where only club members are able to afford publishing openly and pretending that equilibrium constitutes full open access. If enough R1 universities sign agreements like this, it might start to look and feel like open access publishing is a public good supporting open access reading. But that won't be true; people outside that ecosystem will not have the same access to the scholarly dialogue created by publishing openly.

\section{Open access cannot become a club}

If we -- again, the conglomerate of researchers publishing and consuming scholarship - believe in the value of open access, then we should believe and support the public good of open access in all ways, both for reading and for publishing. We should create a system where no individual faces a set of choices that limit their ability to participate in the scholarly dialogue. We should also take a moment to appreciate how far we've come and how much the culture has shifted in order for the idea that readers should pay for scholarly research to appear out-of-touch and inequitable. The scholarly research community has shifted its thinking about the barriers to research that are created when individual readers must find a way to pay, and, for the most part, this community has decided that limiting who has access to reading research limits the quality of research produced.

What we cannot allow to happen is replacing the idea that readers should pay for research with one that says authors should. This is the comfortable notion being offered by transformative agreements that rely upon a pay-to-publish paradigm, and it's one that is gaining traction quickly if the recent news from Cambridge University_ $\underline{\text { Press }}$ is any indication. The idea is attractive because it ostensibly opens up more research for readers everywhere while maintaining the breadth and depth of access upon which institutionally-affiliated researchers have come to rely; it looks like a winwin, and it is for the covered participants. But the repercussions in the wider system are not so beneficent. Yes, more scholarship is now open to readers globally, but both publishers and research institutions are setting up a dynamic where open access publishing has a price that only some can afford to pay. If the idea that authors should pay for scholarly research becomes the new normal, we will not have created a public good called open access. We will have shifted the price of open scholarly publishing from the readers to the authors, and, instead of some readers finding themselves 
turned away at the gates, it will be the authors of new scholarship who will find their paths to open access publishing foreclosed.

Thankfully there is another way, one most frequently labeled diamond open access, that makes the public goods framework of open access an explicit part of the process. $\underline{8}$ For diamond open access journals, it is free to both read and publish. Since it is not actually free to do anything in life, these journals do require support to maintain their infrastructure and editorial staff, but that support does not come from fees from readers or writers but from some third party, or, more likely, some group formed of third-parties. Models like the Open Library of Humanities and the Free Journal Network are leading the way with fully diamond open access portfolios while other groups like the American Mathematical Society. are experimenting with diamond open access with new journals like Communications of the American Mathematical Society.

Diamond open access looks like a true public good, and that's not just because it does not place any burden on readers or authors. Like a public good, diamond open access exists because the publishers who believe in the value of this model are consciously creating public goods. Public goods are not created by spontaneous collective action; they require intent and, most notably, an acceptance on the part of the creators that they will not be able to control the benefits or somehow earn "their fair share" -- there is no idea of a fair share with a public good because there is no sharing. Everyone gets as much as they need without threatening the consumption of anyone else. Diamond open access, like a classic public good, relies on a model where individual incentives do not need to be aligned for the greatest benefit to be created. Instead, they depend upon the beneficence of someone or something that can sustain the public good despite its enormous costs and uncapturable value.

Every time a new transformative agreement is struck, it looks like a win-win for the publisher and the institution. Institutions, currently paying to both read and publish in many cases, can consolidate costs and focus their payment streams on the publishing side, liberating article after article published by their scholars for readers everywhere. Publishers continue to receive nearly as much in revenues, and the upside is in their favor as these transformative models rely upon paying to publish so as more research is published, more will be paid to the publishers. Universities' budgets will feel less chaotic, and, with the burden of open access publishing decisions falling to individual researchers, the option should be much more enticing, especially to groups who have previously been unable to afford the requisite APCs. But standing 
there, just outside the gates, are all the people who still cannot afford the APCs, and, instead of focusing on ways to lower the barriers or demolish them entirely, we're focused on building better clubs for our researchers. And clubs are great, but they are not public goods. And I want the real thing.

Ashley Jester can be found on Twitter (@ashleyrjester)

\section{Footnotes}

1.

Ostrom (2003) provides a thorough review of collective action and public goods.

Ostrom, Elinor. 2003. "How Types of Goods and Property Rights Jointly Affect Collective Action." Journal of Theoretical Politics 15 (3): 239-70. https://doi.org/10.1177\%2F0951692803015003002

2.

Allahar (2017) provides insights into the disruptions that digital publishing and open access have introduced. Johnson et al. (2018), writing in the fifth edition of the STM report provide an overview of scholarly publishing in the digital era, including a section on production costs.

Allahar, Haven. 2017. "Academic Publishing, Internet Technology, and Disruptive Innovation.” Technology Innovation Management Review 7 (11): 47-56. https://doi.org/10.22215/timreview/1120

Johnson, Rob, Anthony Watkinson, and Michael Mabe. 2018. "The STM Report: An Overview of Scientific and Scholarly Publishing." Fifth Edition. https://www.stmassoc.org/2018 10_04_STM_Report_2018.pdf

$\Xi$

3. Besley and Ghatak (2001) look at the benefits of public goods and the need driving their creation as a guide to understanding who should "own" public goods and take responsibility for their costs.

Besley, T., and M. Ghatak. 2001. "Government Versus Private Ownership of Public Goods." The Quarterly Journal of Economics 116 (4): 1343-72. https://doi.org/10.1162/003355301753265598 
4.

Rand et al. (2014) argue that the cost structure facing individuals creates highbarriers to action, preventing public goods formation, and that incentives need to be shifted in order to generate cooperation.

Rand, David G., Erez Yoeli, and Moshe Hoffman. 2014. "Harnessing Reciprocity to Promote Cooperation and the Provisioning of Public Goods." Policy Insights from the Behavioral and Brain Sciences 1 (1): 263-69. https://doi.org/10.1177/2372732214548426 5.

Morgan and Tumlinson (2019) show that for-profit corporations can supply public goods but that doing so requires sacrificing shareholder value in the traditional sense.

Morgan, John, and Justin Tumlinson. 2019. “Corporate Provision of Public Goods.” Management Science 65 (10): 4489-4504. https://doi.org/10.1287/mnsc.2018.3137 6.

Burchardt (2014) looks at the effect on Danish researchers of variations in APC charges, finding that researchers without access to institutional support or waivers were less likely to publish.

Burchardt, Jørgen. 2014. "Researchers Outside APC-Financed Open Access: Implications for Scholars Without a Paying Institution." SAGE Open 4 (4): 2158244014551714. https://doi.org/10.1177/2158244014551714 7.

Fischbacher and Gachter (2010), building upon evidence that individual support of public goods wanes over time, find experimental evidence to support that this finding is generated because individuals prefer the solution where they get to free ride.

Fischbacher, Urs, and Simon Gächter. 2010. "Social Preferences, Beliefs, and the Dynamics of Free Riding in Public Goods Experiments." American Economic Review 100 (1): 541-56. https://doi.org/10.1257/aer.100.1.541

8. 
Jason Kelly (2013) provided an excellent and evergreen overview of open access models.

Kelly, Jason M. 2013. “Green, Gold, and Diamond?: A Short Primer on Open Access.” Jason M. Kelly (blog). January 27, 2013. https://jasonmkelly.com/jason-mkelly/2013/01/27/green-gold-and-diamond-a-short-primer-on-open-access/ 